\title{
The Application of Occasional Color
}

\author{
Yunhan Yu \\ Hangzhou Wanxiang Polytechnic, Hangzhou, Zhejiang, 31000
}

Keywords: occasional color; fabric pattern; design; application

\begin{abstract}
With the continuous development of the market economy, fashion design has a higher color requirements in the fabric. It not only needs to design on the pattern, also needs to ensure that the fabric in the color of the overall effect. The study of occasional color has an important effect on improving the overall effect of the fabric pattern. The occasional color can change the design of the fabric pattern from the two-dimensional plane to the three-dimensional space, resulting in the change of style, the effect, and the formation of new colors. In the process of continuous exploration, the occasional color has a new understanding and the so-called occasional color refers to the process of distribution in the plane showing the effect of free fabric pattern and a color change. Chinese garment industry made a great contribution. This paper will be related to the concept of occasional color in detail, and the application effects of occasional color in fabric pattern design are described, so as to continuously improve the level of fashion design.
\end{abstract}

\section{Introduction}

Often refers to the occasional color creative work in the process of creation of the color of the work due to external factors involved, resulting in the work of color changes, the color of the free hair showing a level of stacking, image dissipation and uneven distribution, The color characteristics of the openness is particularly evident. The use of time and space characteristics to change the color of the past, making the fabric pattern more casual, the use of time and space characteristics of the color changes in the past to make the fabric pattern is more casual, it showing the characteristics of modern abstract art [1]. The so-called sporadic temporal and spatial features generally refers to the shape of the color of the module to be superimposed and the mutual penetration of different colors, to the pattern fabric has brought a richer content. Therefore, the occasional study of color in the fabric design process plays an important role. To a large extent, the rich patterns of design themes and styles promote the rapid development of Chinese fabric and garment industry.

\section{Overview of Occasional Color}

Color in the traditional process of development of the types of restrictions, with the continuous development of society, the occasional color in the process of continuous inquiry made a great degree of progress in the practical application of the research process to promote Chinese garment industry fast Development [2]. In addition, the occasional color gradually from the irregular to the law to follow the direction of the future for the color of the study has laid a good foundation.

In the course of the research, the occasional color is supported by Chinese traditional color, and the idea of western color is integrated into it. The phenomenon of random coloring of random color is studied carefully [3]. In the course of constant exploration of color, the occasional color break through the shackles of the traditional concept of color, forming a new color research idea, is conducive to the apparel industry to enhance the application of color effects.

Occasional color in the course of the study, refers to the creators in the process of color development will be superimposed on the use of different colors to promote the free color of hair growth, changing the original color Mo Yang, reflecting the characteristics of uneven distribution , Belonging to the open color [4]. All of the paintings are free of color on the border, the screen appears more freedom, which is a typical feature of Chinese occasional color and in the whole color 
of the research process has a high Practical value. Occasional color is also a modern abstract art to a certain extent, the occasional color to break the fixed form of the past, in a specific time and space, the effective design of the results of color hair, break the permanent color of the principle, Freedom of color concept.

In the course of research on occasional color in the West, it is found that occasional colors are more distinct in color than traditional Chinese colors, and are easily accepted by the public. The color of the whole chapter has little connection with the overall shape of the design. In the process of appreciation, it needs to use abstract thinking angle to try to figure out, making the pattern more rich and full [5]. The use of occasional colors in the fabric pattern can be used to make different types of color interaction between the mutual interference of the free color is its final result, making the fabric pattern is more abundant in color, only the boundary with a fuzzy Ontology color, showing a diversity.

\section{The Innovative Design of Occasional Color}

Occasional color in the process of continuous inquiry found a lot of features, occasional color itself is the result of free hair color, is largely a combination of natural phenomena, the color in the form of integration with nature, in line with popular aesthetic Of the requirements [6]. Color in the process of development through the use of natural phenomena, making the occasional color in the research process has made great progress.

Creator in the course of research on the occasional color, the color is actually the creator of inner feelings of catharsis, the color can express the inner feelings of people and blue to a large extent expressed the inner sense of depression. In addition, Red reflects the heart of the fiery people [7]. In the course of the study of the occasional color, the color of the effective integration can reflect the creator of the mood.

In the process of exploration, the unconscious stage of color is directly raised to the practice stage, and the occasional color changes the randomness and creation of the past in the course of long-term use. In the use of the process can feel the color of the use of value [8]. Occasional color for the color of the fabric pattern provides an important reference to a large extent, to improve the development of the apparel industry, but also to promote our country on the occasional color of further research.

Occasional innovative design in the research process has important significance is to determine the occasional color and color of the innovative methods of research, can effectively enhance the overall level of occasional color research, so the fabric pattern in the application process to form New forms, the development of the apparel industry has an important significance [9]. The application of occasional color in long-term practice, the improvement of innovation level, the change of the past color hysteresis, occasional color from a certain extent can also be controlled by subjectivity, through practice, the formation of occasional color in the process of Regular, continuous color innovation, resulting in more occasional color, to ensure that the fabric pattern of color diversity.

Accidental color in the usual circumstances, are the result of accidents, there is no artificial creation, the color of the discovery is mainly through the color sensitivity of the creator, from accidentally obtained color. Therefore, the creators of color through detailed study to create more fabric color, the color will be widely applied to the process of costume design, can effectively enhance the overall level of fashion design. Occasional innovative design can be a reasonable study of color and the creators in the design process can use computer technology for a reasonable color design to ensure the overall effect of the fabric pattern, while reducing the waste of manpower and material resources, thereby reducing resources of the waste [10]. In addition, the occasional innovative design is to speed up the color of the study, to cultivate the designer's thinking, to enhance the overall effect of clothing, showing a higher value. 


\section{The Application of Occasional Color in Fabric Pattern}

With the rapid development of market economy, the development of garment fabric pattern in the process of color requirements more and more widely, the economic development makes people's aesthetic level is also rising and the color of clothing people have a higher demand. Therefore, it is necessary to speed up the study of sporadic color, break the traditional color mode, and promote the diversity of clothing colors [11]. Occasional color in the form of more freedom, to reflect the harmony and unity of nature, in the study of the fabric pattern is more humane, in line with the development needs of contemporary society.

The application of sporadic color in the pattern of the fabric has a variety of applications in printing, embroidery and other fields more widely, printed fabrics in the fabric pattern with occasional color, so that in the aesthetic ability has been greatly improved [11]. In addition, the occasional color in the field of weaving flowers are more, weaving itself in the shape has its own unique, it is difficult to fill the color pattern of fabric, and occasional color to break through this drawback, making woven flowers The color appears to be more full.

Occasional colors in the fabric design process have an important position. The development of modern technology to the occasional color design provides a good technical support. Accidental color in the process of innovation need to explain the conditions in detail, do a good job on the relevant statistical data for the future with color reference. Computer database, constantly updated development, this provides a very good platform. In addition, the study of the occasional color need to refer to the process of science and technology, computer applications will be color control in a certain range, to ensure that the color pattern design process in the fabric showing good results, can greatly reduce the probability of failure [12]. In the course of design, the occasional color has a huge space for development. Through long-term practice, we can get rich color reference value. In the course of designing, applied to the fabric pattern, thereby enhancing the overall effect. In the process of designing, it can not only improve the efficiency of design, maximize the advantage of occasional color, but also ensure the quality of the product. It can be used in the process of using random color data statistics, color simulation control and color reference value analysis. , Reduce the defective rate.

Spontaneous germination is the main feature of occasional color, but also an important process of color innovation, before the advent of occasional color and media intervention can significantly enhance the color of the scientific nature of the formation of the color of the systematic form. Infrequent color innovation model presents the basic characteristics, in many areas of the application process have gained great success, widely applied to various fields of color, especially in the field of fashion design applications, to enhance the apparel industry of the overall development.

\section{Conclusion}

In summary, the occasional color is created by the creator inadvertently, not under the control of creator and makes free germination from colors, which presents a variety of colors, thereby enhancing the fabric pattern integrity effect. From the occasional color layering, the color through the overlay, cover and other forms continue to form a new color, making the fabric pattern to show diversity in the color. In addition, the application of sporadic color in the design process of fabric pattern can change the pattern from two-dimensional plane to three-dimensional plane and reflect the richness of color change, which has an important influence on the design of garment fabric pattern. Therefore, the occasional color researchers need to strengthen the study of the relevant color, from the daily practice to constantly sums up experience, so as to promote the rapid development of Chinese entire garment field.

\section{References}

[1] Song Jingyuan, Wang Yuqi, Yao Sidian, Liang Liefeng. Application of pattern design in modern 
textiles [J]. Journal of Chengdu Textile College, 2016, 01: 186-189.

[2] Zou Hui, Cheng Bingying. Analysis of modern children's clothing market adult and its impact on children's design inspiration [J]. Modern Silk Science and Technology, 2016, 01: 31-34.

[3] Sun Xuemei. "non-legacy" Fujian Putian Matsu costume language and its cultural connotation [J]. Art and Design (Theory), 2016, 04: 126-128.

[4] Xu Hong, Yan Wenjun. Studies on Women's Wear in the Western Regions of the Tang Dynasty and Reflections on the Design of Contemporary Women's Wear - A Case Study of Xinjiang Unearthed Cultural Relics [J]. The Silk, 2016, 06:58-64.

[5] Wang Qi, Lei Ye, Li Zhenying, Sun Rui, Fan Xinwang, Xu Lanbing. Symbolic Application of Peking Opera Elements in Fabric Printing Design [J]. Art and Design (Theory), 2016, 06: 88-90.

[6] Jin Shuang, Mu Xuemei.Application of "Batik dyeing" digital printing technology in creative design of clothing [J]. Modern Decoration (Theory), 2016,07: 83.

[6] Wang Hui, Zeng Qi, Guo Ying, Wei Jianyu, Wei Yisu. Innovative application of leather fabric secondary design in luggage design [J]. Leather Science and Engineering, 2014, 06: 58-62.

[8] Wang Zengcheng, Zhao Fanghua. Analysis of Tang Dynasty dress elements and its application in modern evening dress design [J]. Sichuan Drama, 2014, 12: 123-125.

[9] Qiao Wen, Chen Xiaocheng, Xue Sunan.Chinese traditional blue calico secondary creative costume design expression [J]. Textile Industry and Technology, 2015,02: 46-48.

[10] Wang Jinmei, Yan Yannong, Wu Xiaochen, Zhang Bin, Zhang Xuepei, Xu Dongliang.Design of digital printing pattern of cashmere fabric imitated cross stitch [J]. Knitting Industry, 2013, 11: 35-38

[11] Zhang Jian. Interpretation of fish grain modeling in the secondary design of fabric heritage and innovation [J]. Shandong Textile Economy, 2013, 04: 62-63 +83.

[12] Han Jianlin. Based on Fujian Province under the consumption of sports shoes segmentation stylized design analysis [J]. Shandong Textile Science and Technology, 2013, 04: 48-51. 